\title{
EXPERIENCIA CON EL MODELO AMPLIADO DE DISEÑO AXIOMÁTICO EN PRODUCTOS DE APOYO QUE FAVOREZCAN LA INCLUSIÓN SOCIAL
}

\author{
Jaime Aguilar-Zambrano \\ Doctor en Proyectos de Ingeniería e Innovación \\ Facultad de Ingeniería. \\ Pontificia Universidad Javeriana, Cali \\ jaguilar@javerianacali.edu.co
}

\author{
Diana Riveros \\ Estudiante de Maestría Int. Enviroment and \\ Resources Management ENREM, \\ Universidad Autónoma de San Luis Potosí, México \\ Cologne University od Applied Sciences, Alemania \\ dirospina@hotmail.com
}

\author{
Claudia Marcela Sandoval \\ Estudiante Especialización en Diseño y Gerencia de \\ Producto para la Exportación, \\ Pontificia Universidad Javeriana, Bogotá \\ claumasa@gmail.com
}

\section{Resumen:}

En este artículo se describen las estrategias utilizadas por el equipo del proyecto de investigación ANDAR, en la experiencia con el Modelo Ampliado de Diseño Axiomático (MADA) de Aguilar-Zambrano (2009), durante el proceso de creación de un producto de apoyo a la movilidad que favoreciera la inclusión social de personas en situación de discapacidad.

El MADA, como método prescriptivo de trabajo, permite que el equipo de diseño trabaje de manera interdisciplinaria, propiciando la convergencia de representantes de la universidad, la empresa, el Estado y el usuario, alrededor de una necesidad social. De ahí que propicie una estructura de desarrollo de producto centrada en el usuario, pues es quien la conoce en profundidad. Esta forma de abordar la problemática plantea diferencias fundamentales con enfoques exclusivos del mercado o de la academia; sin embargo, no riñe con ninguno de los dos.

De la experiencia de trabajo con el MADA, se concluyó que es una propuesta adecuada para el desarrollo de productos en equipos que busquen resolver necesidades sociales, pues se tiene un marco común de referencia que enfoca el proceso. También se encontró que la comunicación entre disciplinas y las cualidades personales de los miembros del equipo tienen alta incidencia en el proceso de diseño, y se evidenció la importancia del contacto directo de las profesiones con el usuario, para que éste participe en todas las decisiones que determinen el rumbo del proyecto.

El proyecto de investigación ANDAR se realizó en Cali, Colombia, entre 2009 y 2010, gracias a la financiación del Departamento Administrativo de Ciencia, Tecnología e Innovación Colciencias.

Palabras Clave: Diseño de producto, Trabajo en equipo, Diseño Axiomático, TRIZ, Producto de apoyo, Interdisciplinariedad, Modelo Ampliado del Diseño Axiomático, Discapacidad, Silla de ruedas. 


\begin{abstract}
:
In this article are described the strategies used by the team of research ANDAR, in the experience with the extended model of axiomatic design (MADA) of Aguilar-Zambrano (2009), during the process of creation of a product of support to the mobility that helps the inclusion of persons in incapacity situation.

The MADA, as a prescriptive method of work, allows that the design team works in an interdisciplinary way, encouraging the convergence of representatives of College, the company, the State and user, around a social necessity.

The research project ANDAR was made in Cali, Colombia, between 2009 and 2010, thanks to the financing of theDepartamentoAdministrativo de Ciencia, Tecnología e Innovación.
\end{abstract}

Keywords: product design, work in team, axiomatic design, TRIZ, support product, interdisciplinary, extended model of axiomatic design, incapacity, wheelchair.

\title{
Introducción
}

El proyecto ANDAR nació de la necesidad de verificar la efectividad del modelo ampliado de diseño axiomático (MADA) que desarrolló J. Aguilar-Zambrano (2009), cuyo núcleo es resolver una necesidad social. El MADA es un complemento a la propuesta metodológica del diseño axiomático de N. P. Suh (1990), a través de la inclusión de manera explícita de los requerimientos subjetivos o no funcionales de un producto (NFRs: Non FunctionalRequirement), los cuales se diferencian de los requerimientos técnicos clásicos (FRs: FunctionalRequirements). De manera adicional, sugiere la conformación de un equipo de diseño interdisciplinar y multi-organizacional (usuario, empresa, universidad y Estado); y por último, propone el uso de la teoría de solución de problemas inventivos (TRIZ), como técnica para el análisis sistémico de problemas y para la generación de ideas dentro de la fase creativa del proceso de diseño.

El MADA está compuesto por tres partes: la primera, se enfoca en la conformación del equipo de trabajo; la segunda, abarca el análisis sistémico del problema y el primer nivel del diseño; y la tercera, comprende los niveles inferiores y la verificación de los dos axiomas del diseño axiomático. Dentro de estas tres partes del MADA se aplican herramientas de TRIZ, una teoría propuesta por Altshuller (1946), y del diseño axiomático de Suh (1990).

El proyecto que aquí se describe empleó el marco de trabajo propuesto en el MADA en la creación de un producto de apoyo a la movilidad, con el fin de favorecer la inclusión social de las personas en situación de discapacidad motriz. Para este propósito se tomaron como referencia los productos de apoyo a la movilidad que se encuentran clasificados según la norma ISO9999:2007 (Aenor, 2007) dentro de la clase 12 que incluye, entre otros, a los ciclos, las sillas de ruedas y los ciclomotores.

En el artículo se describe la experiencia y las estrategias de trabajo utilizadas por el equipo del proyecto ANDAR durante el desarrollo del producto, el cual culmina en el diseño de una silla de ruedas bipedestadora. Para la verificación del MADA el equipo contó con autonomía durante el desarrollo de las tres partes del modelo descritas anteriormente.

El artículo se encuentra organizado de la siguiente forma: la primera parte describe los antecedentes del proyecto, donde se definen el MADA, el diseño axiomático y TRIZ; la segunda parte presenta las tres partes constitutivas del MADA, en las que se detallará en el funcionamiento de las mismas y se expondrá la experiencia del desarrollo del producto de apoyo en el proyecto ANDAR; a continuación, se presenta el resultado del proceso con el diseño del producto y, por último, las conclusiones derivadas después de un proceso de reflexión al final del proyecto. 


\section{Antecedentes de la experiencia de diseño: MADA, diseño axiomático y TRIZ}

\section{- Modelo Ampliado del Diseño Axomático (MADA)}

El MADA (Aguilar-Zambrano, 2009) se basa en la propuesta del diseño axiomático (Suh, 1990), al que adiciona la determinación de la necesidad social como centro de convergencia para el equipo de diseño, lo cual permite que las organizaciones participantes puedan ejercen un rol más efectivo dentro del modelo. También se incluyen los requerimientos subjetivos de un producto o requerimientos no funcionales (NFRs: Non FunctionalRequirements), diferenciándose de la visión clásica de requerimientos técnicos o requerimientos funcionales (FRs: FunctionalRequirements) del diseño axiomático. Por último, el MADA también involucra un equipo de diseño interdisciplinar y favorece el proceso creativo con la aplicación de TRIZ (Salamatov, 1999). La Figura 1 muestra la composición conceptual del MADA.

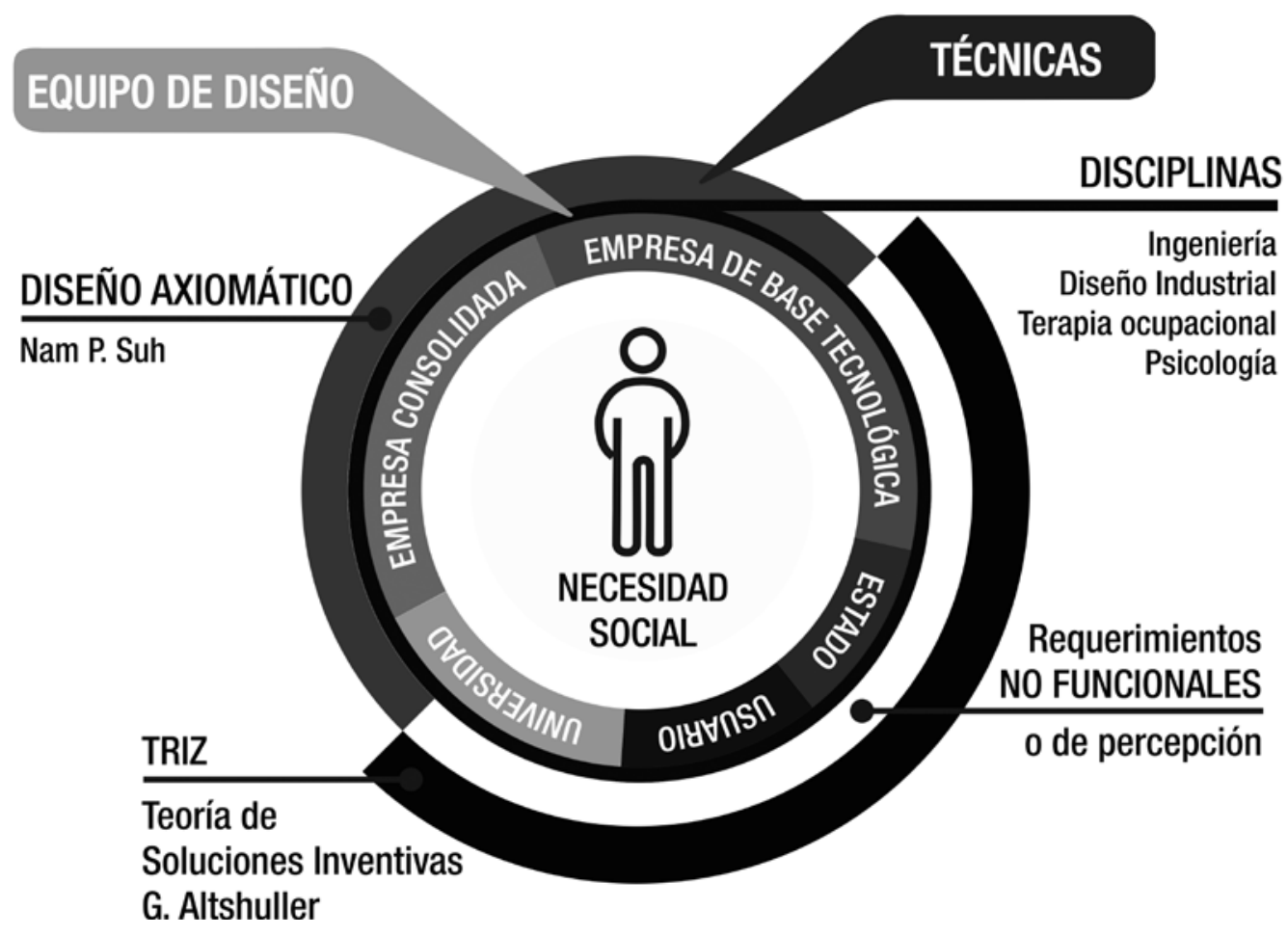

Figura. Composición del MADA: El equipo de trabajo que tiene como convergencia la necesidad social, y técnicas que lo soportan, el diseño axiomático con adición de requerimientos no funcionales y TRIZ.

El MADA consta de tres partes como se muestra en la Figura 2. La primera es la conformación del equipo de trabajo, la segunda es el análisis sistémico del problema, en donde se determina una estructura jerárquica del diseño; y la tercera parte comprende la estructuración del proceso de diseño para los niveles inferiores o de detalle. Allí se verifica permanentemente el cumplimiento de los dos axiomas que define el diseño axiomático. 


\section{- Diseño Axiomático}

La propuesta de Suh (1990) define una estrategia para el diseño de productos acompañada de una base científica. En esta estrategia se presentan cuatro dominios que funcionan como se muestra en la Figura 3. Con base en dicha figura, en el dominio del cliente, las necesidades de los usuarios se traducen como los Atributos del Cliente (CAs: Client Atributes). En el dominio funcional, estos atributos se convierten en un conjunto de requerimientos funcionales (FRs: FunctionalRequirements). En el dominio físico se escoge un conjunto de parámetros de diseño (DPs: DesignParameters) que son soluciones técnicas para satisfacer dichos Requerimientos Funcionales que vienen determinados por unos rangos de diseño (DRs: DesignRanks), por fuera de los cuales un determinado parámetro de diseño no se considera satisfactorio. Por último, en el dominio de proceso se selecciona un conjunto de variables del proceso (PVs: ProcessVarables) que determinan cómo se materializarán los parámetro de diseño del dominio físico.

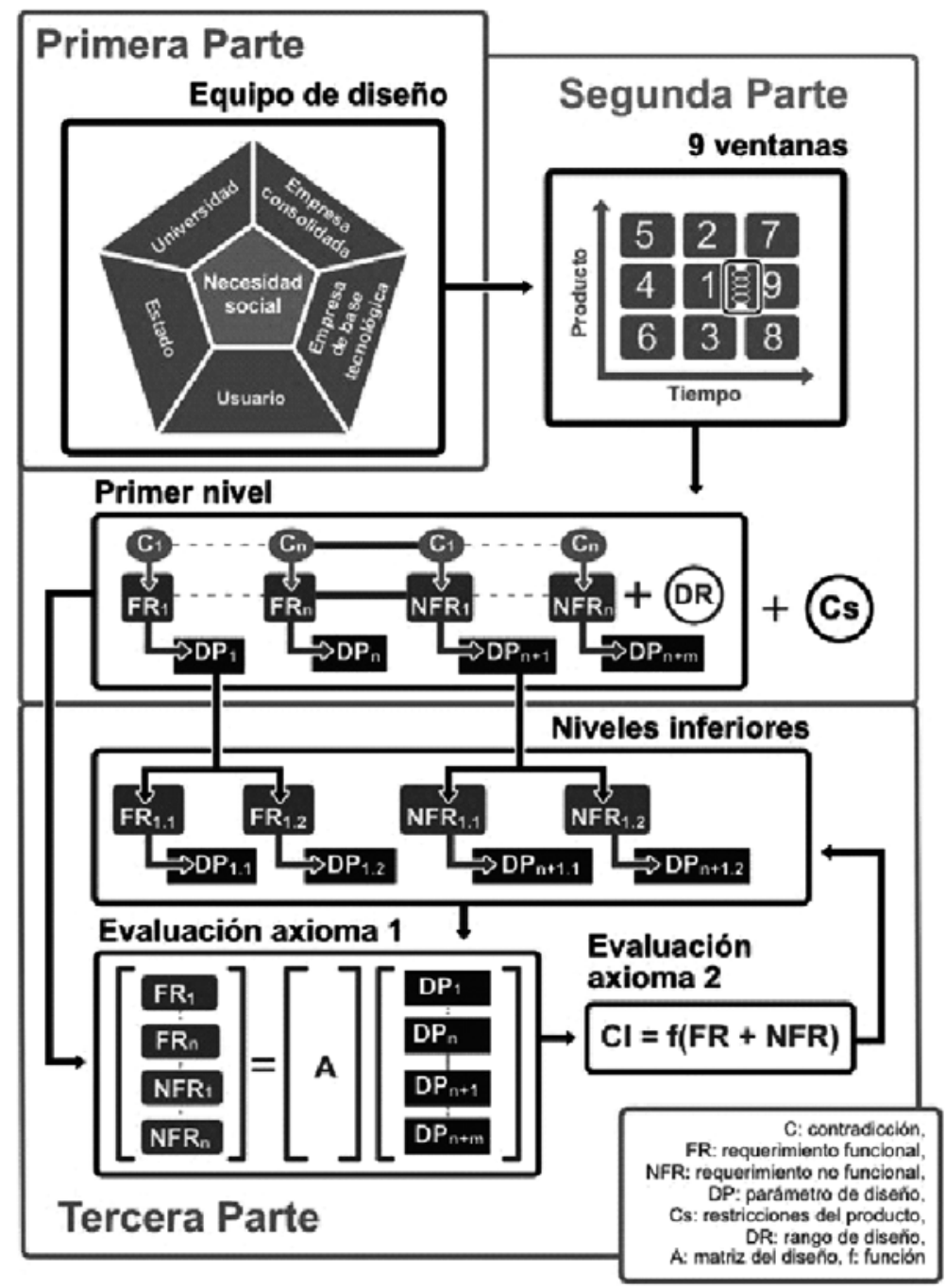

Figura. Partes del MADA: El equipo de diseño con sus cinco actores, el análisis de las nueve ventanas, la estructuración del primer nivel y los niveles inferiores, y los dos axiomas del Diseño Axiomático. 


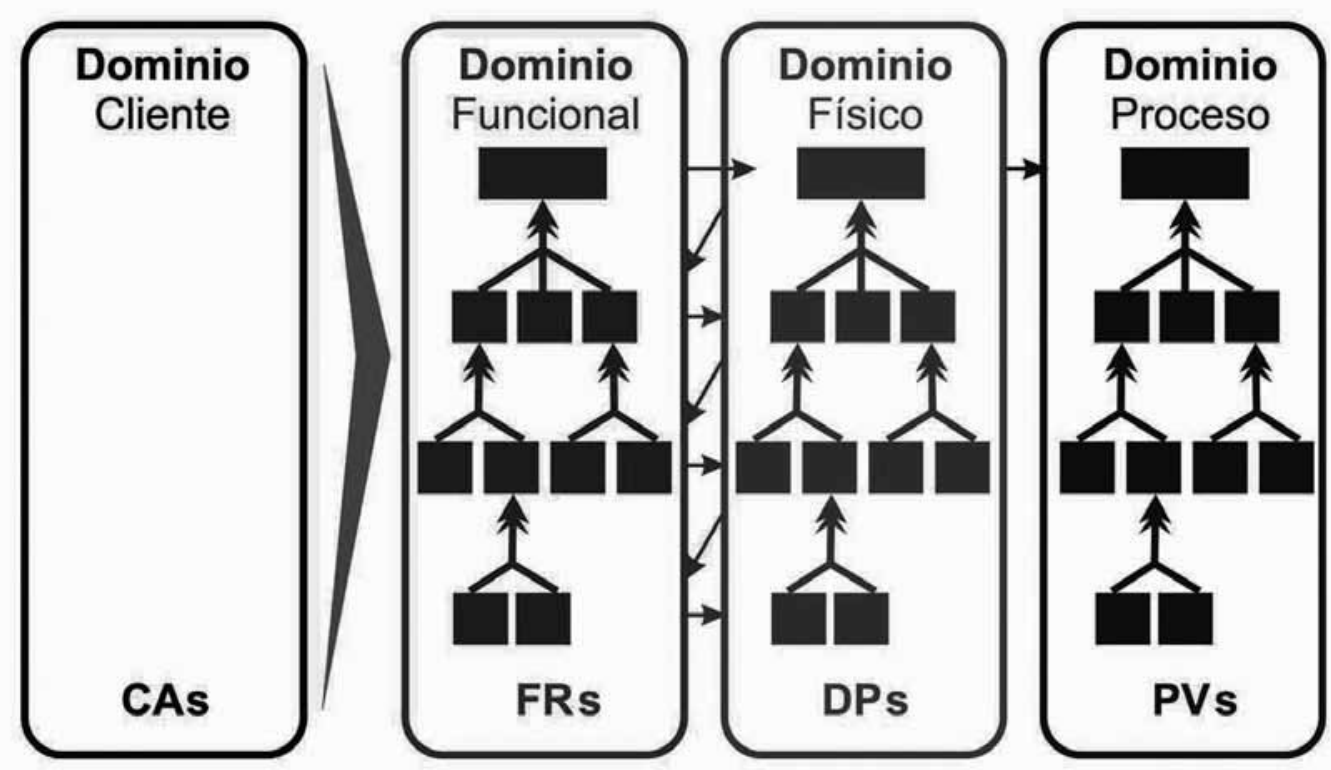

Figura. Dominios definidos por Suh (1990) en su propuesta original del diseño axiomático.

\section{- Los dos axiomas en el diseño axiomático}

Este método recibe su nombre de la aplicación de dos axiomas que sirven para garantizar la calidad del diseño, estos son: el axioma de independencia y el axioma de información. El axioma de independencia afirma que durante el proceso de diseño, la relación mapping o zigzagueo entre requerimientos funcionales y parámetros de diseño, se debe establecer de una forma tal que una perturbación en un parámetro de diseño particular afecte solamente a su requerimiento funcional asociado. $\mathrm{Al}$ mismo tiempo, el axioma de información establece que, entre todos los diseños que satisfacen el axioma de independencia, el mejor diseño es aquel con el menor contenido de información.

Matemáticamente, la ecuación de diseño que expresa el axioma de independencia, se enuncia de la siguiente manera:

$$
\left\{\begin{array}{c}
F R_{1} \\
F R_{2} \\
F R_{3} \\
\vdots \\
F R_{m}
\end{array}\right\}=\left\{\begin{array}{cccc}
A_{11} & A_{12} & \ldots & A_{1 n} \\
A_{21} & A_{22} & \ldots & A_{2 n} \\
\vdots & \vdots & & \vdots \\
A_{m 1} & A_{m 2} & \ldots & A_{m n}
\end{array}\right\}\left\{\begin{array}{c}
D P_{1} \\
D P_{2} \\
D P_{3} \\
\vdots \\
D P_{n}
\end{array}\right\}
$$


Por su parte, el contenido de información (I) se puede definir cuantitativamente como el logaritmo de la probabilidad (p) de satisfacer el requerimiento especificado, como se observa en la Ecuación 2. El contenido de información es cero cuando el desempeño del sistema (dado por el rango del sistema) está siempre dentro del rango de variación permitido (dado por el Rango del Diseño).

Ecuación. Contenido de información

\section{$I=\log \_2(1 / p)$}

\section{- Teoría de Solución de Problemas Inventivos (TRIZ)}

TRIZ es una teoría desarrollada por Altshuller en 1946 (Savransky, 2000), que se compone por un conjunto de técnicas que ayudan al diseñador en la fase creativa del diseño. La teoría parte de la siguiente hipótesis: existen principios universales de invención que cuando se identifican y codifican, hacen que los procesos inventivos se vuelvan más predecibles y sirvan como base para obtener innovaciones que favorezcan el avance de la tecnología. Los conceptos básicos de TRIZ son: idealidad, contradicciones y evolución de los sistemas técnicos.

\section{- Diseño del Producto de Apoyo: Conceptos y Aplicación del MADA}

A continuación se describe la aplicación del MADA en el diseño de un producto de apoyo, según sus tres partes.

\section{- Primera Parte del Modelo Ampliado del Diseño Axiomático}

En esta parte se clarifica la necesidad social y se conforma el equipo de diseño de naturaleza interdisciplinar en torno a ella.

\section{- La necesidad social en el MADA.}

El MADA plantea que el punto de convergencia del equipo interdisciplinar no debe ser solamente un problema de mercado y tampoco uno exclusivamente científico o académico, sino una necesidad social, la cual tiene implicaciones desde el mercado y requiere para su solución del conocimiento científico. 


\section{- La necesidad social en el proyecto ANDAR}

En este paso, un equipo de trabajo preliminar la definió como: El favorecimiento de la inclusión social de personas en situación de discapacidad, por medio del diseño de un producto de apoyo.

Debido a que existen múltiples productos de apoyo, fue necesario realizar un proceso de toma de decisiones multicriterio y multiexperto con la estrategia AnaliticHierarchyProcess o análisis jerárquico AHP (Saaty, 1994), para seleccionar un producto de apoyo específico que estuviera contemplado dentro de la norma ISO9999 (Aenor, 2007). Los resultados obtenidos, que se muestran en la Figura 4, determinaron que el producto a desarrollar debería encontrarse entre la clase 12 de la norma, es decir, sería un producto para asistir la movilidad personal. La Figura 5 muestra una representación de los productos de la clase 12.

A partir de esta clarificación de la necesidad social, se procedió a formar el equipo de diseño que trabajaría durante el proyecto y que complementaría al equipo preliminar.

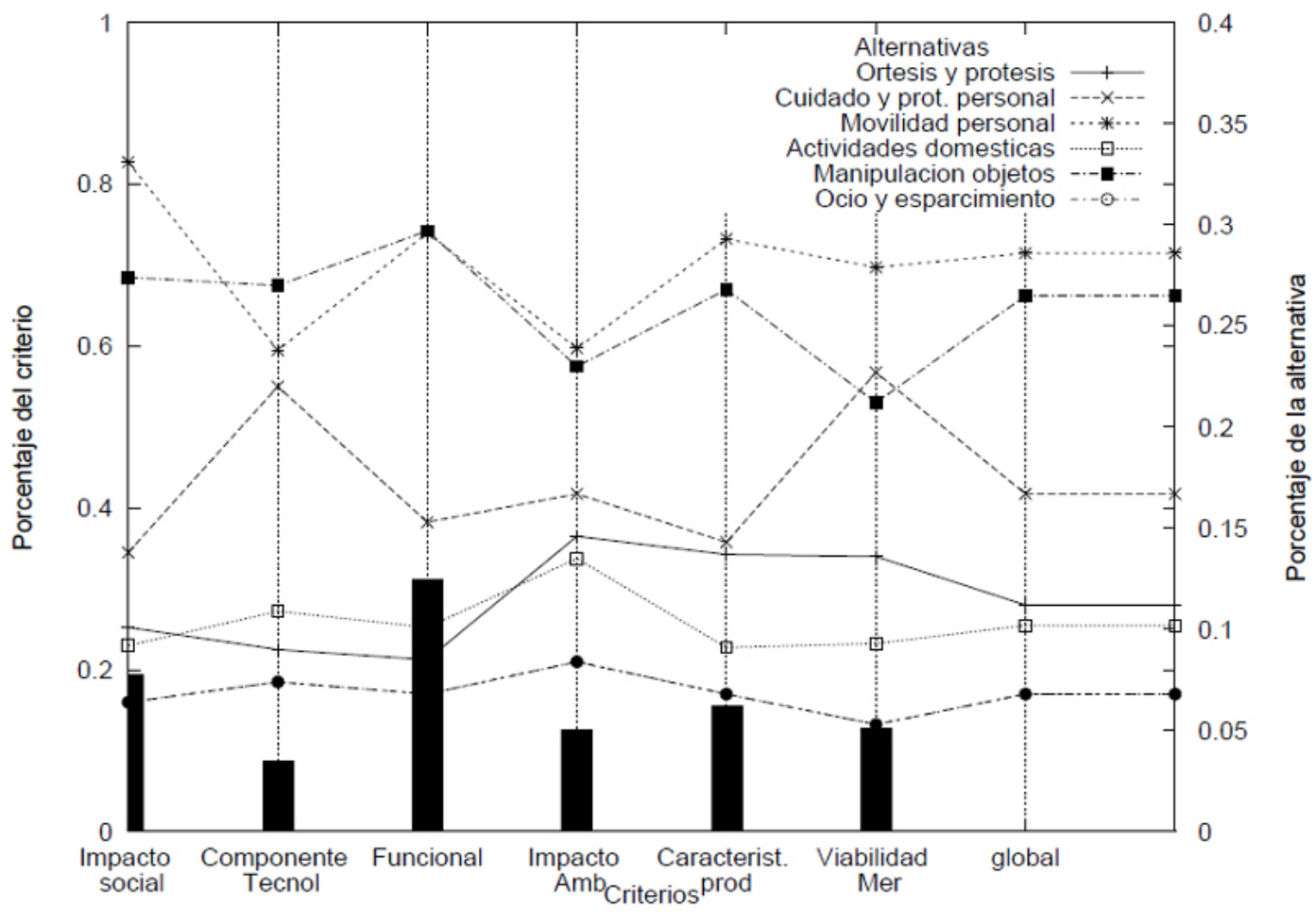

Figura. Resultados del uso de AHP para seleccionar un producto que debe ser objeto de diseño 


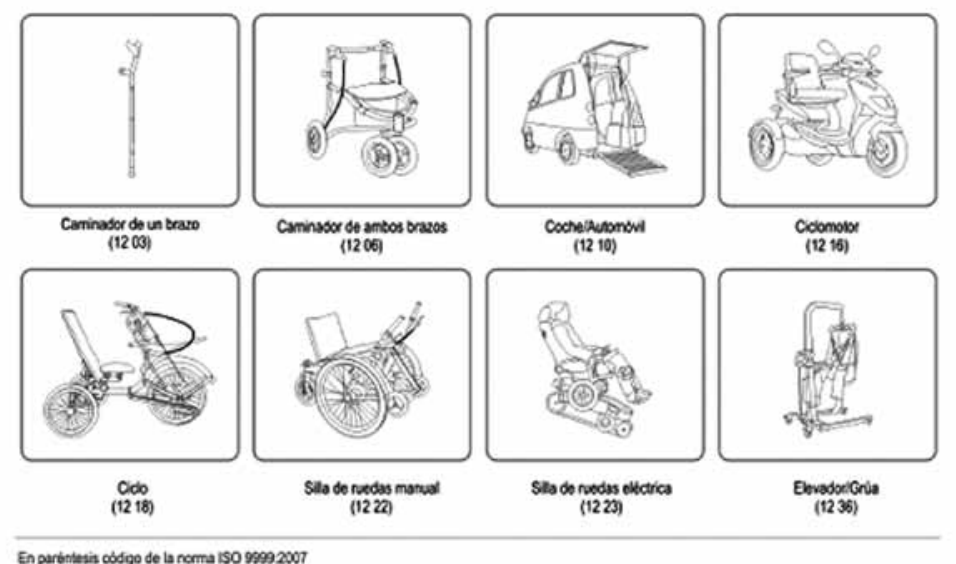

Figura. Productos de apoyo a la movilidad de la clase 12 de la norma ISO9999

\section{- El equipo en el MADA}

El MADA plantea que desde el aspecto organizacional el equipo se debe conformar con cinco actores representados por personas de diferente procedencia disciplinar, asociados a la problemática de interés. Esta interacción, entre personas y entidades, tiene varias ventajas como la ampliación del campo de búsqueda de soluciones y el intercambio de saberes y experiencias. En la Figura 6 se observa la dinámica de interacción entre los diferentes actores y sus aportes, que también se detallan seguidamente:

- Universidad: profesionales de diferentes áreas del conocimiento con incidencia en la necesidad social (se sugiere la presencia permanente de personal de ingeniería y diseño industrial).

- Empresa consolidada: con la participación de las áreas de mercadeo, diseño e ingeniería de producción. Entidad experta en la necesidad social y con vínculos fuertes hacia la industria y el mercado.

- Empresa de base tecnológica: que debe ser experta o afín a la necesidad social; con vínculos fuertes hacia la técnica, la ciencia y la academia.

- Estado: instituciones públicas, relacionadas con la necesidad social, que se vinculan con personas o con apoyo económico para financiar proyectos.

- Usuario: una persona o un conjunto de ellas, que se seleccionan a partir de un estudio de la necesidad social.

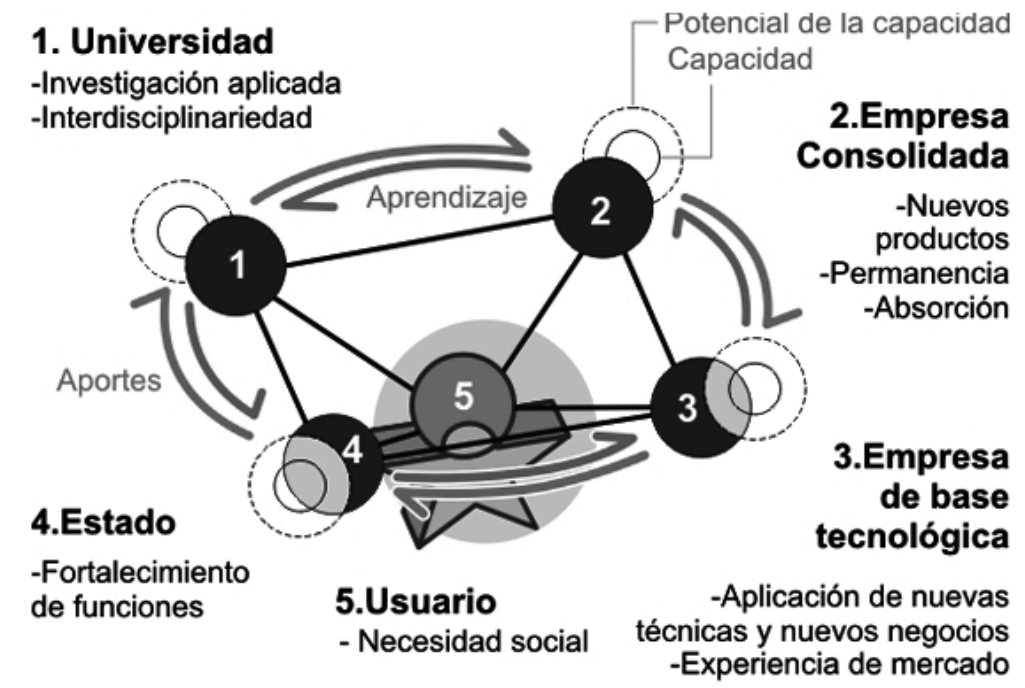

Figura. Equipo de diseño en el MADA se configura de acuerdo al modelo de colaboración universidad-usuario-empresa. Basado en el trabajo de Aguilar-Zambrano, J. GonzalezCruy\& Aguilar-Zambrano J.J. (2009). 


\section{- El equipo en proyecto ANDAR.}

Desde el punto de vista organizacional el equipo se integró de la siguiente manera:

El usuario, que se representó por miembros de instituciones de personas en situación de discapacidad: Club Nuevos Horizontes y la Gran Alternativa, separados en dos grupos, uno amplio de 45 personas, y de éste, un subgrupo seleccionado. La participación del primer grupo fue emitir opinión en entrevistas y encuestas, mientras que la participación del segundo grupo se especificó en grupos focales y sesiones de trabajo. En este subgrupo, la presencia varió entre uno a ocho individuos, dependiendo de la actividad a realizar.

El Estado se representó con el Departamento Administrativo de Ciencia, Tecnología e Innovación, Colciencias y la Secretaría de Bienestar de la Alcaldía de Cali; La Universidad: con profesionales de la Pontificia Universidad Javeriana-Cali, la Universidad del Valle y la Universidad Politécnica de Valencia. La empresa de base tecnológica con Rehabitec Ltda. No se contó con la presencia de una empresa consolidada en el equipo porque no existen en el país empresas que diseñen y construyan este tipo de productos.

Desde la perspectiva de disciplinas, los profesionales pertenecían a las áreas de ingeniería eléctrica, electrónica, mecánica e industrial; diseño industrial, terapia ocupacional y psicología. En la Figura 7 se muestra esta participación en tres niveles. El investigador principal lideró y garantizó el seguimiento del MADA, mientras que el círculo de investigadores mayores tomó decisiones basadas en su experiencia y en la información recopilada por el círculo de jóvenes investigadores, quienes realizaron el grueso del trabajo investigativo. La selección de este recurso humano incluyó el test de personalidad Myer-Briggs (Shen et al., 2007).

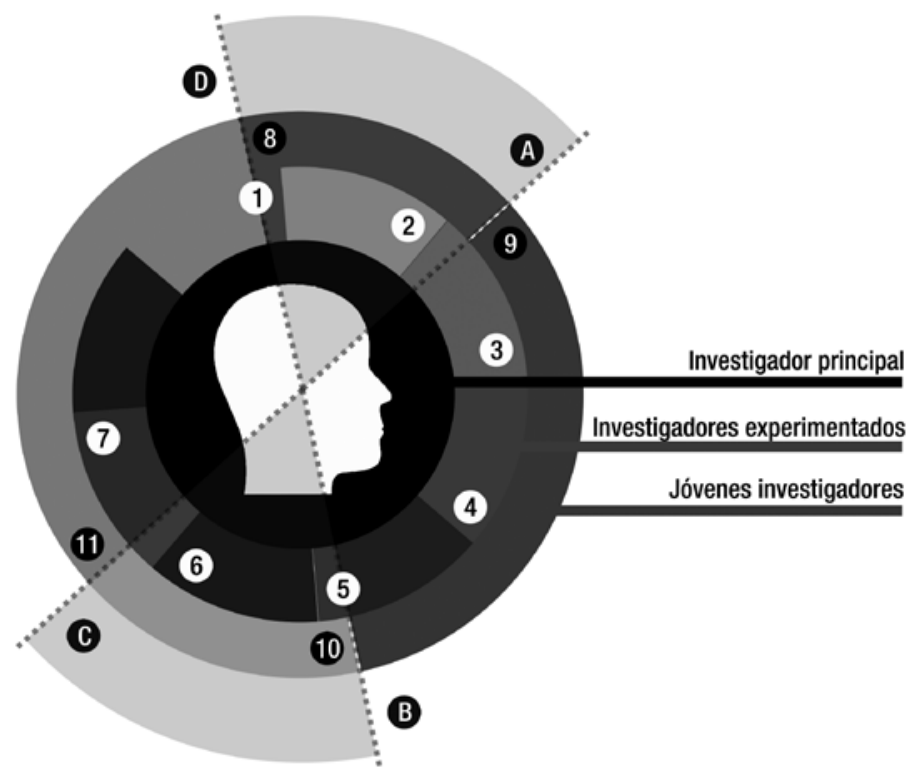

COMPOSICIÓN DISCIPLINAR DEL EQUIPODEINVESTIGACIÓN:

Investigador principal: Ingeniería eléctrica/ Investigadores experimentados: 1. Ingenieria mecánica (x2), 2. Ingeniería eléctrica, 3. Psicología, 4. Terapia ocupacional, 5. Diseño Industrial, 6. Ingeniería electrónica, 7. Ingeniería industrial/ Jóvenes investigadores: 8. Ingeniería electrónica, 9. Diseño Industrial (x2), 10. Ingeniería industrial, 11. Ingeniería mecánica (x2).

A, B, Cy D:Zonas diferenciadas de mayor interacción entre investigadores jóvenes y experimentados.

Figura. Composición disciplinar e interacción del equipo de investigación del proyecto ANDAR 
Finalmente, el equipo base resultante se compuso de un investigador principal, ocho investigadores experimentados y seis jóvenes investigadores, para un total de 15 participantes. Cabe anotar que a lo largo del proyecto se generaron dinámicas espontáneas de trabajo por subgrupos, según la afinidad de disciplinas o de experiencias sobre las actividades a ejecutar.

\section{- Segunda parte del Modelo Ampliado del Diseño Axiomático}

Esta parte comprende el análisis del problema y formulación del primer nivel del diseño axiomático.

\section{- Análisis del problema en el MADA}

El MADA deja a criterio del equipo la forma de efectuar este análisis, pero sugiere utilizar técnicas de TRIZ como el análisis de evolución de los sistemas técnicos y las nueve ventanas.

Por su parte, el análisis de evolución se basa en los paradigmas: (a) todo sistema técnico se compone de cuatro partes: motor, transmisión, herramienta y objeto (Figura 8) que le sirven para transformar la energía de entrada en una función principal útil para un objeto determinado, y (b) para alcanzar la idealidad, el sistema técnico debe evolucionar con el paso del tiempo. Entonces, para determinar el estado del proceso evolutivo de un sistema técnico, se puede evaluar su nivel de cumplimiento de las Leyes de Evolución propuestas por Altshuller (CavalluccI y Weill, 2001), lo que a su vez sirve para identificar oportunidades de innovación futuras.

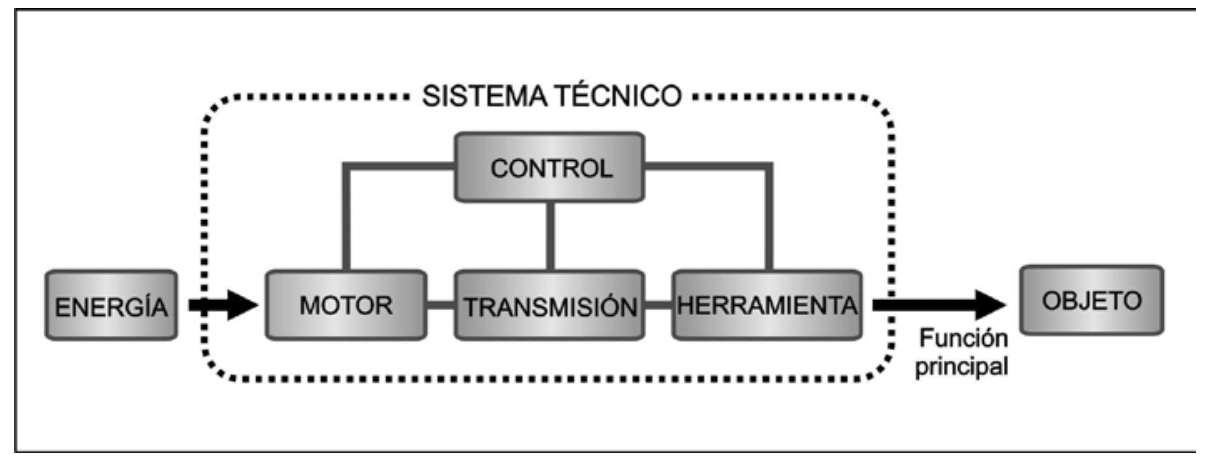

Figura. Representación de las partes de un sistema técnico. Basado en Cavalluci (2009)

De otro lado, la técnica de las nueve ventanas permite realizar el análisis sistémico del problema a partir de la función principal de un sistema técnico y lo proyecta al futuro (Savransky, 2000). Esta técnica consiste en la descripción y análisis del sistema, su entorno (súper-sistema) y sus componentes (sub-sistemas) tanto en el presente como en el pasado y el futuro, lo que se puede visualizar en una matriz de $3 \times 3$. Cabe anotar que mientras el presente y el pasado son descritos con base en hechos reales, el futuro es imaginado por el equipo de diseño en la búsqueda de la idealidad del sistema. Como resultado se obtienen patrones evolutivos del producto y se detectan contradicciones técnicas que impiden alcanzar ese futuro hipotético. 


\section{- Análisis del problema en el proyecto ANDAR}

En el proyecto ANDAR, el análisis del problema se realizó desde las perspectivas social, técnica, normativa y del mercado. A continuación se detalla el proceso:

\section{Perspectiva social o análisis del usuario}

Con el fin de identificar requerimientos básicos de los productos de apoyo a la movilidad personal de la población en situación de discapacidad, el equipo de trabajo realizó: (a) una revisión documental de estadísticas y reportes finales de estudios realizados en el Valle del Cauca por las secretarías de salud departamental y municipal junto con datos del DANE, (b) entrevistas a representantes de las instituciones de y para la atención a la discapacidad en Cali, (c) la aplicación de un primer instrumento para la clasificación de la personas según características funcionales, y (d) la aplicación de un segundo instrumento para la recolección de información de la población en situación de discapacidad motora, con el fin de caracterizar del usuario por actividades, participación y relación con el entorno.

\section{Perspectiva técnica o análisis del producto}

Para efectuar este análisis se realizó: (a) un análisis de evolución de los sistemas técnicos, y (b) un proceso de vigilancia tecnológica.

En la Figura 9 se muestra, por medio de diagramas de radar, el estado evolutivo de los diferentes productos de la clase 12 (véase Figura 5). Este análisis arroja un indicio de cuáles son los productos más y menos evolucionados, y determina algunos conceptos claves para el proceso de vigilancia tecnológica.

Imagen Diagramas de radar

Figura. Análisis de la evolución técnica de productos de apoyo, según diagramas de radar. El polígono externo representa la situación ideal, y el interno, la situación actual. 
En el proceso de vigilancia tecnológica se revisaron productos comerciales y artículos científicos y se analizaron patentes de productos de apoyo de la clase 12.

Una de las dificultades para el equipo de diseño, en esta etapa, radicó en entender la complejidad del acople entre un sistema natural humano que hace parte integral de un sistema técnico, ya que los productos de apoyo no son ajenos a la personas, sino una extensión de ellas, debido a una deficiencia en el sistema natural original. Así pues, este tipo de sistema se denominó como sistema técnico híbrido y se así consideró para futuros análisis dentro del proyecto.

\section{Perspectiva normativa o análisis de estándares}

Como el diseño del producto debe cumplir con los estándares internacionales que le conciernen, se realizó un análisis de las normas relacionadas con la situación de discapacidad, en el que se encontraron las normas específicas para las sillas de ruedas, de ahí que los estándares: UNE 111915:1991, UNE 111913:1991, UNE 111914-11:1995, UNE 12182:2000, UNE 12183:2010, UNE 12184:2010, UNE 26494, UNE 41500, UNE 41510, UNE 41520, UNE 41523, UNE 11191591, UNE 139801, UNE 139802, UNE 170001-1, se utilizaron durante todo el proceso, especialmente durante la selección de los parámetros del diseño.

\section{Perspectiva de mercado o planeación por escenarios}

Desde el punto de vista de inclusión social, la inclusión laboral de las personas en situación de discapacidad fue un aspecto relevante del proyecto. En este sentido, se realizó un estudio con el fin de valorar la viabilidad comercial de los productos de apoyo para la movilidad personal. Este estudio consistió en el análisis de la importancia de los productos, los actores y la cadena de comercialización en el mercado actual y el planteamiento de modelos de negocios. En la Figura 10 se muestra uno de los modelos propuestos, en donde se involucra a las personas en situación de discapacidad, como parte de una cadena de producción en donde ellos son al mismo tiempo usuarios del producto y beneficiarios de la empresa.

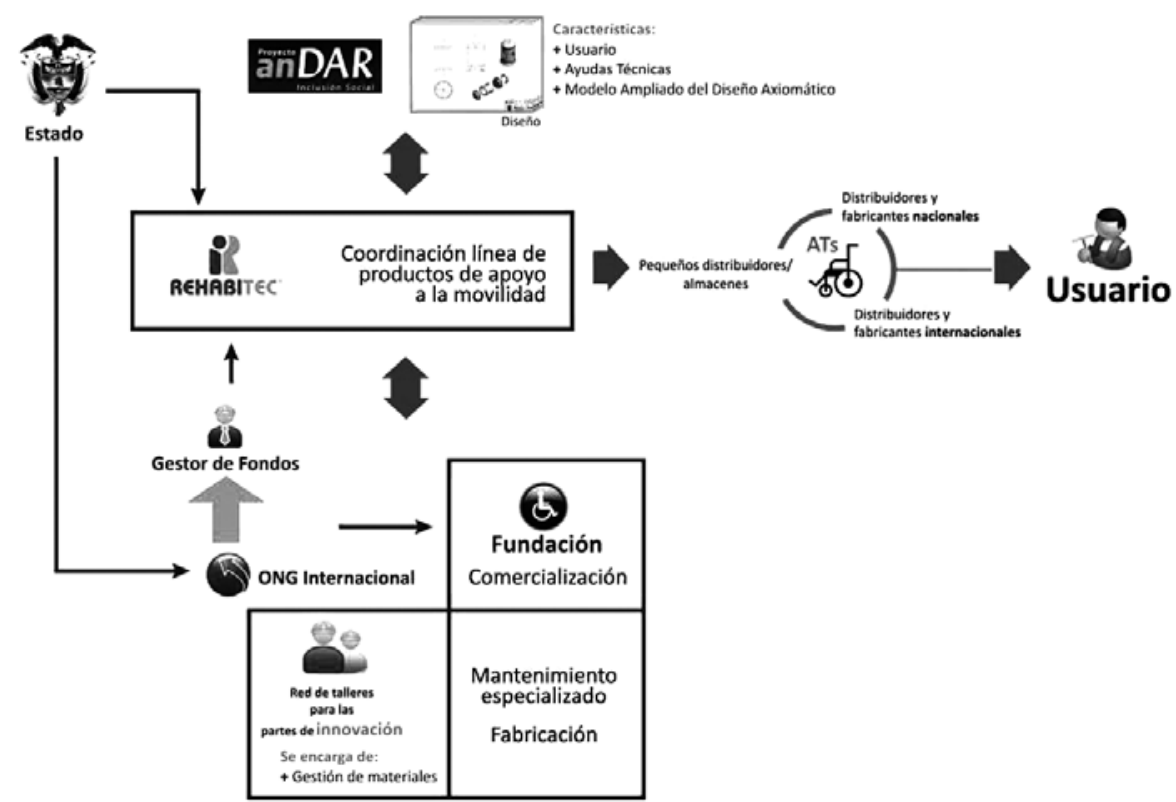

Figura. Modelo de negocio con participación de las personas en situación de discapacidad. 
Con estas perspectivas y un análisis del pasado de este tipo de productos de apoyo, el equipo utilizó la técnica de las nueve ventanas con el fin de definir la idealidad del sistema y encontrar contradicciones técnicas. Las contradicciones técnicas son conflictos que no permiten, en el presente, que el sistema evolucione a su estado ideal.

\section{- Formulación del primer nivel del diseño axiomático en el MADA}

El principal insumo para esta etapa es el análisis de las nueve ventanas, del que se extraen contradicciones (CNs) y necesidades (CAs: Client Atributes). Esta información debe provenir de un trabajo conjunto del equipo de diseño, en donde el usuario es esencialmente quien conoce a profundidad la necesidad social.

La idea del primer nivel es traducir estas contradicciones y necesidades en requerimientos funcionales y no funcionales (FRs y NFRs: Functional\& Non-FunctionalRequirements), cada uno de los cuales deberá encontrar una solución tangible mediante un parámetro de diseño (DPs: DesignParameters). Adicionalmente, dichos parámetros deben encontrarse dentro de los rangos de diseño (DRs: DesignRanks), que son los límites de los requerimientos funcionales asignados por el equipo y que permitirán la posterior evaluación, a partir del axioma de información de las alternativas de solución.

Para encontrar los parámetros más adecuados, el MADA sugiere que el equipo de diseño utilice la matriz de contradicciones, una técnica de TRIZ con el fin de encontrar principios de diseño; son estrategias que han sido previamente exitosas para resolver contradicciones similares. Las restricciones (Cs: Constraints) son limitantes que determinan la selección de los parámetros de diseño. En la Figura 11 se indica cómo funciona esta estructura o árbol de diseño.

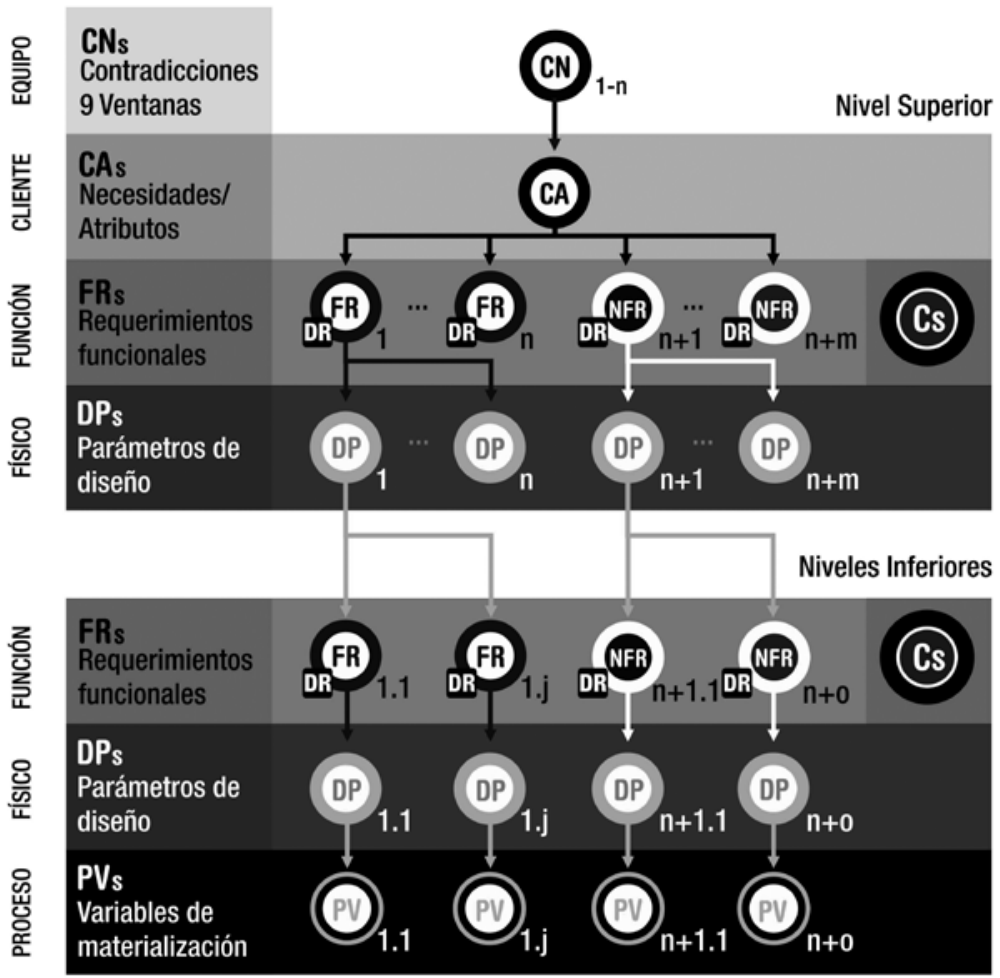


Todos estos elementos deben ser exactos en su definición y reflejar de forma precisa la necesidad y el deseo del usuario, por lo que su participación durante esta etapa es indispensable.

\section{- Formulación del primer nivel del diseño axiomático en el proyecto ANDAR}

Siguiendo la estructura anteriormente descrita, se formuló el primer nivel conjuntamente con el usuario. Durante diferentes sesiones de trabajo se construyó la Tabla 1, la cual recopila los trece requerimientos detectados en esta fase, según las partes del sistema técnico.

Durante el primer nivel también se explicitan las restricciones de diseño (Cs), las cuales se formularon de manera cualitativa, así: 'mantener al mínimo' y 'mantener al máximo', por la dificultad de establecer un valor preciso en la primera visión del problema (Tabla 2).

Tabla. Requerimientos funcionales iniciales para el primer nivel del diseño de un producto de apoyo a la movilidad

\begin{tabular}{|c|c|c|}
\hline \multirow{2}{*}{$\begin{array}{c}\text { Parte del } \\
\text { Sistema técnico }\end{array}$} & \multicolumn{2}{|r|}{ Requerimientos Funcionales (FR) } \\
\hline & Número & Descripción \\
\hline Motor & 1 & Completar la potencia proporcionada por la persona \\
\hline Transmisión & 2 & $\begin{array}{l}\text { Transmitir la energía de la persona para que el sistema } \\
\text { técnico se desplace }\end{array}$ \\
\hline \multirow{4}{*}{ Control } & 3 & $\begin{array}{l}\text { Mantener el equilibrio dinámico del sistema técnico frente } \\
\text { al entorno }\end{array}$ \\
\hline & 4 & $\begin{array}{l}\text { Permitir que el usuario controle el desplazamiento } \\
\text { (dirección) del dispositivo }\end{array}$ \\
\hline & 5 & Permitir que el usuario controle el frenado del dispositivo \\
\hline & 6 & $\begin{array}{l}\text { Permitir que el usuario acceda al mínimo espacio diseñado } \\
\text { para el tránsito de caminantes (corredores) }\end{array}$ \\
\hline \multirow[t]{3}{*}{ Interfaz } & 7 & $\begin{array}{l}\text { Mantener al usuario en una temperatura corporal que no le } \\
\text { genere malestar }\end{array}$ \\
\hline & 8 & Disminuir los puntos de presión que puedan generar escaras \\
\hline & 9 & Permitir que el usuario aborde y abandone el dispositivo \\
\hline \multirow{4}{*}{ Herramienta } & 10 & $\begin{array}{l}\text { Permitir que el usuario se desplace por diferentes } \\
\text { superficies en sentido vertical (trepar: subir escaleras) }\end{array}$ \\
\hline & 11 & $\begin{array}{l}\text { Permitir que el usuario se desplace por diferentes superficies } \\
\text { en sentido horizontal (interacción dispositivo-terreno) }\end{array}$ \\
\hline & 12 & $\begin{array}{l}\text { Permitir la adaptación del dispositivo según el nivel de } \\
\text { lesión del usuario, manteniéndolo en equilibrio }\end{array}$ \\
\hline & 13 & Adoptar las posturas erguido, sedente y semisedente \\
\hline
\end{tabular}




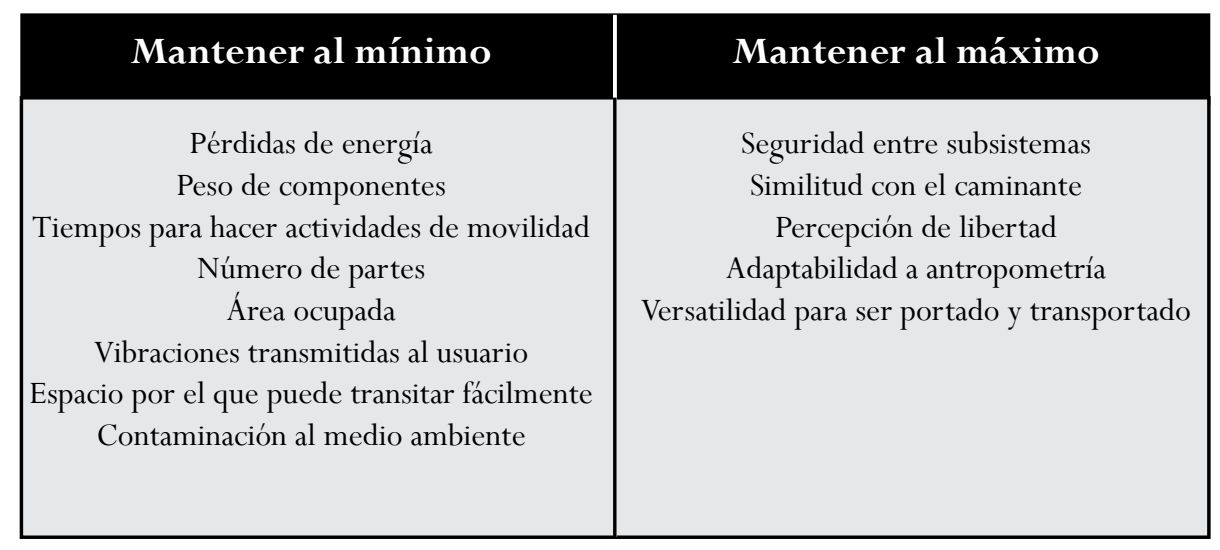

Tabla. Restricciones de diseño (Cs)

Con estos insumos se aplicó la matriz de contradicciones, la cual arrojó principios de diseño que inspiraron al equipo a sugerir una gran variedad de parámetros y, con la combinación de los mismos, plantear nueve alternativas de solución.

Seguidamente, un subgrupo de trabajoque incluyó al usuario definió, mediante un proceso analítico jerárquico AHP, con cuál de estas nueve alternativas se continuaría el proceso. Así, estas personas evaluaron la capacidad potencial de cada propuesta para: (a) subir escaleras, (b) permitir la manipulación del sistema o tener un alto grado de usabilidad, (c) permitir el traslado desde y hacia la silla, y (d) para cambiar de postura. La alternativa seleccionada se planteó gráficamente como se muestra en la Figura 12.
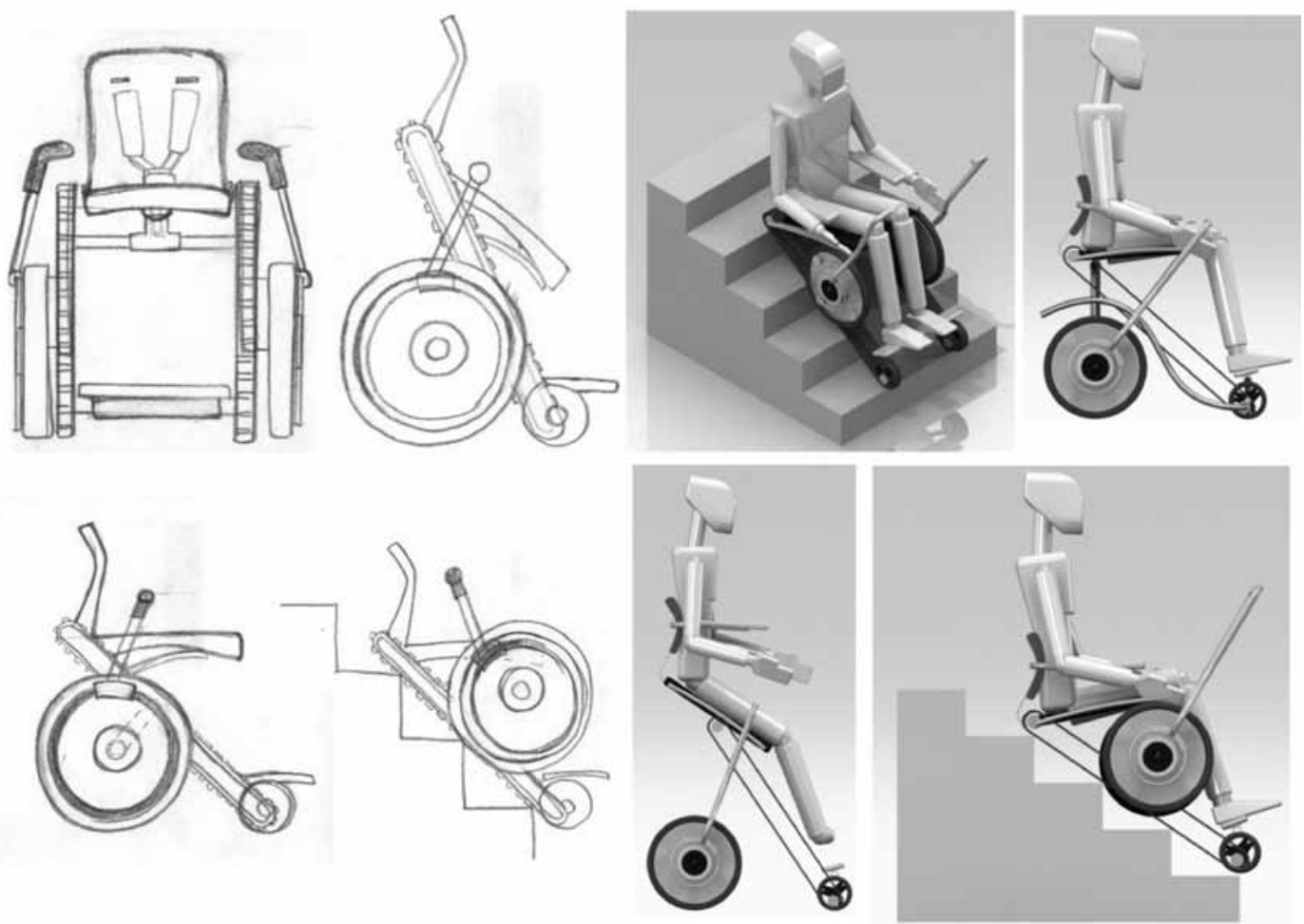

Figura. Representación gráfica de la alternativa mejor valorada por un subgrupo de trabajo que incluyó la participación del usuario para continuar el proceso de diseño. 
Finalmente, después de un cuidadoso análisis de la alternativa seleccionada y su árbol jerárquico de diseño, se determinó que era necesario hacer un replanteamiento en la jerarquía del producto, pues se evidenciaron acoples en los requerimientos funcionales de alto nivel, con lo cual se violaba el axioma de independencia y se dificultaba el establecimiento de los rangos de diseño.

De esta forma, se plantearon nuevos requerimientos de primer nivel como se observa en la matriz del diseño de la Figura 13. La nueva matriz solo tuvo cuatro requerimientos, los cuales se consideraron independientes y además, lograron contener todas las necesidades planteadas la primera iteración.

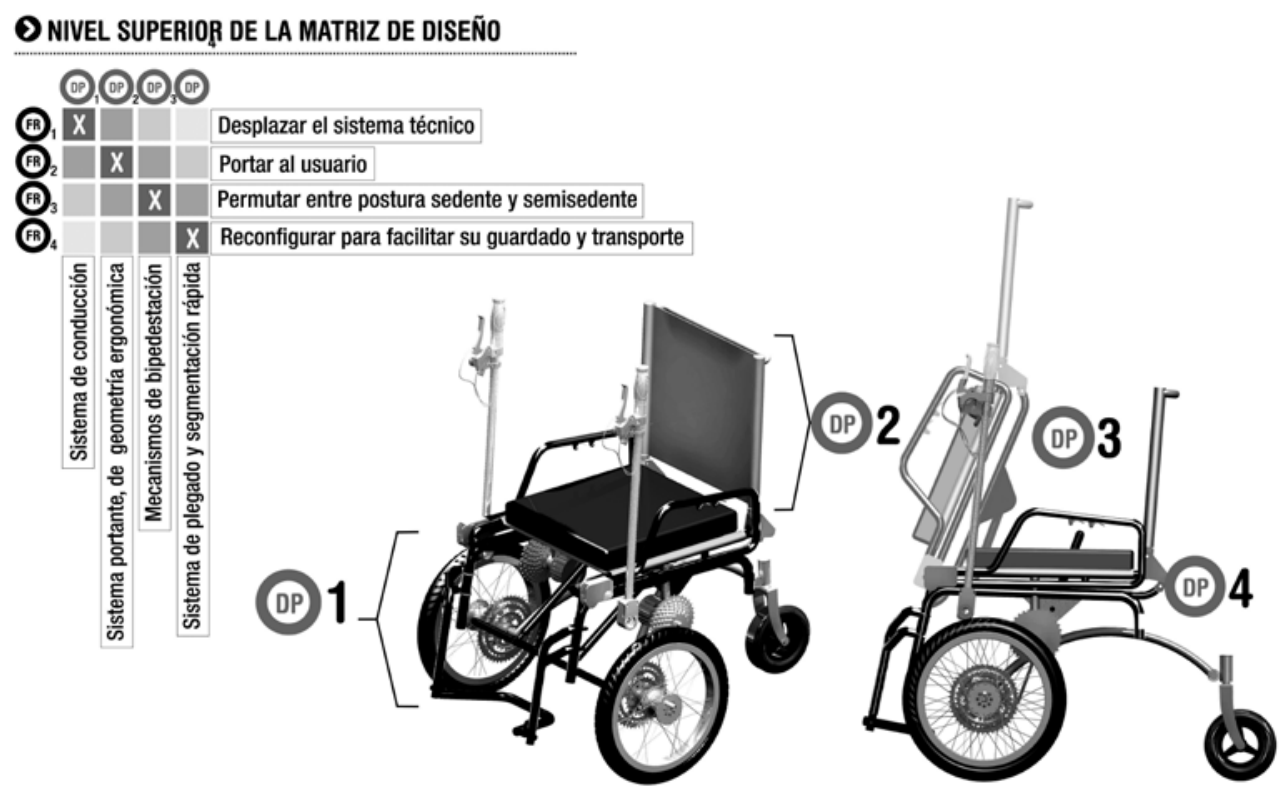

Figura. Matriz del diseño del nivel superior. La nueva matriz logró desacoplar los requerimientos y conservar la información de la primera iteración.

\section{- Tercera Parte del Modelo Ampliado del Diseño Axiomático}

La tercera parte corresponde a los niveles inferiores del árbol jerárquico y evaluación de los axiomas del diseño axiomático, el axioma de independencia y el del contenido de información, para estos niveles.

\section{- Niveles inferiores y evaluación de axiomas en el MADA}

Una vez se completa el primer nivel, se procede al planteamiento de los requerimientos funcionales de nivel inferior, que son aquellas actividades necesarias para satisfacer el parámetro de diseño superior. El resultado de este proceso es una matriz compleja, pues cada parámetro genera nuevos requerimientos que deben ser satisfechos para llevar a cabo la propuesta de diseño. El último nivel, generalmente de carácter técnico, describe con detalle hasta la más pequeña pieza de un producto o el más pequeño elemento de un proceso. 
Al mismo tiempo, se debe verificar el cumplimiento de los axiomas del diseño axiomático. De un lado, en el axioma de independencia, deben procurarse el menor número de acoplamientos funcionales posibles entre requerimientos y parámetros. Una vez se plantean todos los parámetros de diseño, se procede a determinar las variables de proceso, que no son más que formas de manufacturar dichos parámetros.

De otra parte, para la verificación del axioma de información, se efectúa el cálculo del contenido de información medida indirecta de la complejidad de un diseño el cual, entre mayor sea, mayor es la complejidad de un diseño, y también, cuando se alcanza un diseño con un mínimo contenido de información, significa que el problema de apariencia compleja pudo ser resuelto de manera efectiva.

\section{- Niveles inferiores y evaluación de axiomas en el proyecto ANDAR}

En este proyecto se usó el software Acclaro $_{\mathrm{TM}}$ tanto para la verificación del axioma de independencia, como para el cálculo del contenido de información. La Tabla 3 muestra el contenido de información para cada requerimiento, usando una distribución de probabilidad uniforme.

Una de las ventajas de proponer las alternativas de solución a partir de la matriz de contradicciones de TRIZ, es que la matriz de diseño resulta desacoplada, situación que no se puede garantizar cuando se plantea una solución global con requerimientos funcionales directos del usuario.

\begin{tabular}{|c|c|c|c|c|c|} 
FR & \multicolumn{4}{|c|}{ Rango } & Contenido \\
Ce Información \\
a
\end{tabular}

Nota. Unidades: abits, ${ }^{\mathrm{b}} \mathrm{m} / \mathrm{s}$, ${ }^{\mathrm{c}} \mathrm{kg}$, ${ }^{\mathrm{d}}$ grados, ${ }^{\mathrm{e}}$ porcentaje de reducción de volumen

Tabla. Contenido de información de los requerimientos funcionales del primer nivel

De acuerdo con estos cálculos se infiere que el contenido de información del sistema es 1,958. Este nivel es un buen indicador de la calidad del diseño puesto que un diseño óptimo ideal tendría un valor igual a cero, lo cual significaría que los parámetros de diseño cumplen en su totalidad con las exigencias de los rangos de diseño. 


\section{- Resultado del proceso}

El resultado de la aplicación del MADA se observa en la Figura 14. Se trata de una silla de tres ruedas, manual y bipedestadora, que se impulsa por medio de palancas.
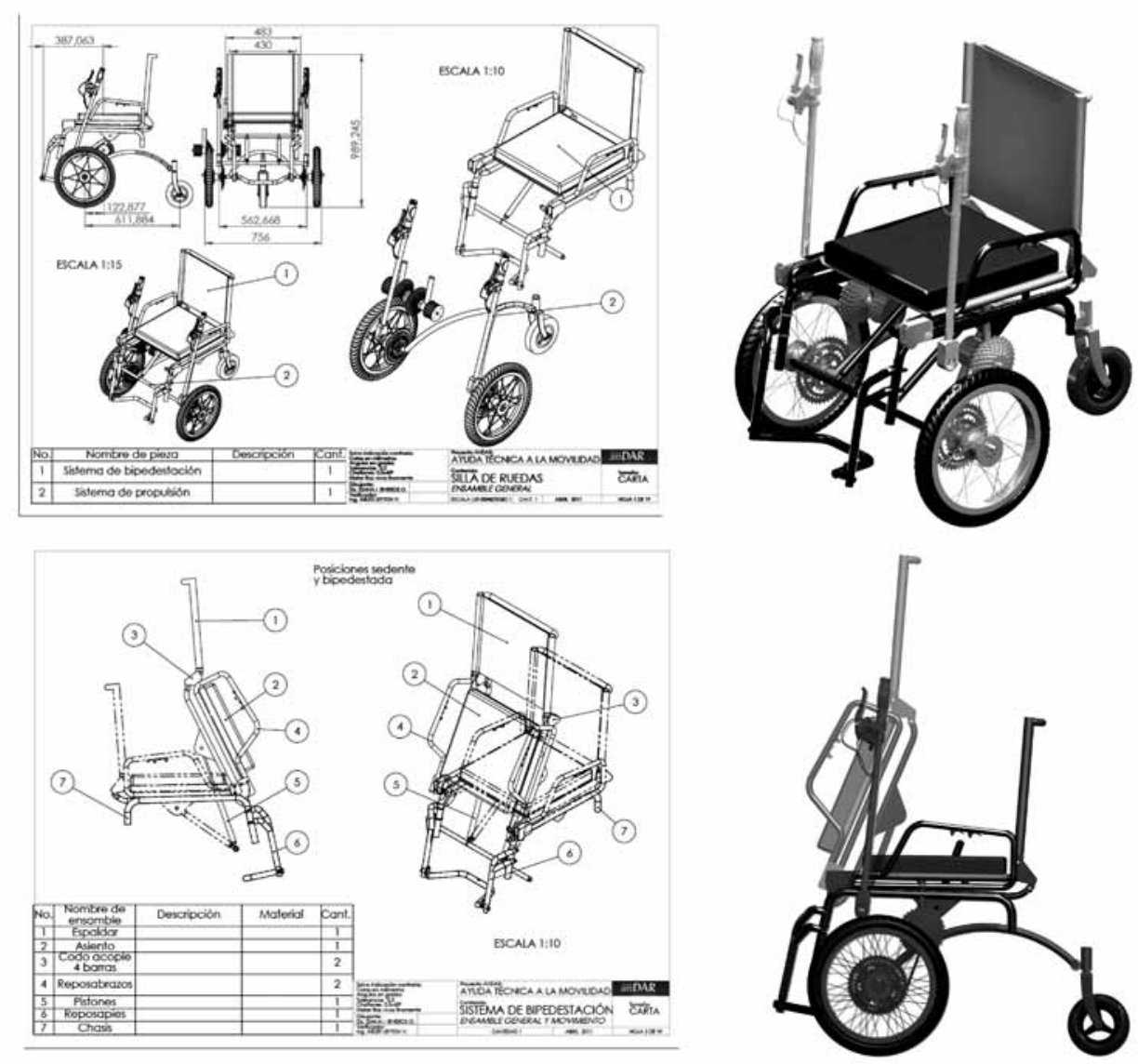

Figura. Producto de apoyo a la movilidad personal. Resultado del proyecto de investigación ANDAR

Toda la energía que requiere proviene del usuario, lo que la hace más liviana y versátil que una silla motorizada; y gracias a los mecanismos de los que dispone, el usuario puede sentir menos fatiga que con una silla de impulsión por aro. Las palancas, las ruedas y la estructura en general permiten que el usuario se desplace por diferentes terrenos y con diferentes niveles de esfuerzo.

Las ruedas son más pequeñas que las de las sillas de ruedas convencionales pues se hace innecesario el aro de empuje y permiten que la silla sea más liviana y fácil de maniobrar. La tracción delantera sirve para que el usuario suba bordillos de una manera más fácil y rápida y la rueda trasera sirve para la estabilidad al maniobrar y, especialmente, durante la bipedestación.

Las palancas, además de aumentar la eficiencia en la trasmisión de la fuerza, evitan que el usuario sufra lesiones en las articulaciones de los miembros superiores por las malas posturas al aplicar fuerza en el aro, y al mismo tiempo, fomentan la actividad física. Estos movimientos que pueden ser alternados o de remo se efectúan en rangos ergonómicamente correctos.

La bipedestación se controla desde las palancas y los reposabrazos, para bajar y subir, respectivamente. El descenso se hace por fases, a la velocidad que el usuario pueda mover las palancas, y la bipedestación o ascenso se hace por medio de un mecanismo neumático más rápido para que el usuario alcance la posición semisedente en corto tiempo. 


\section{Conclusiones}

El MADA es una propuesta adecuada para el trabajo de diseño con equipos interdisciplinares porque permite tener un marco común de referencia para el proceso de diseño.

La literatura presenta el trabajo entre disciplinas como una estrategia adecuada para obtener buenos resultados, sin embargo no existen experiencias empíricas suficientes que permitan dar fe de esta situación, lo que hace de este proyecto un precursor en su clase. Se concluye que este tipo de experiencias debe ser fomentado desde la academia para tener una visión amplia sobre el campo de diseño de producto. De esta forma, se promueve la generación de espacios de encuentro entre disciplinas, con el fin de que el joven profesional reduzca la brecha en la comunicación, y no ocasione sesgos disciplinares ni auto-exclusión en los procesos de trabajo en equipo. Sin embargo, es necesario trabajar en la formación de grupos interdisciplinares para el diseño de productos con el fin de aumentar la efectividad del equipo, pues se evidenció que conformar el equipo por medio de estrategias de fundamento psicológico no es garante de eficiencia, ya que la capacidad de trabajo en equipo debe desarrollarse a partir de estrategias que lo motiven durante el proceso de diseño. Para ello es imperativo realizar una preparación previa al trabajo de diseño, probablemente bajo acompañamiento psicológico, y contemplar un tiempo para esta actividad dentro del plan del proyecto.

El proyecto fortalece las teorías del diseño centrado en el usuario, lo cual debe ser un proceso orientador en todos los procesos de diseño. El contacto directo con el usuario amplía la visión del problema, en especial de las disciplinas más ajenas a la necesidad social, y sensibiliza en torno a ella. Por lo tanto, es recomendable que todo el equipo participe en los procesos de recolección de datos, previo entrenamiento. De igual forma, el usuario debe participar en etapas del proceso en las que se tomen decisiones que tengan alta incidencia en el rumbo del proyecto.

186 Se requiere mayor número de experiencias de aplicación del MADA puesto que en este proyecto se encontró que después del estudio a fondo de la necesidad social, algunos requerimientos que habían sido identificados como no funcionales o subjetivos, podían ser traducidos a funcionales o técnicos. De igual manera, se evidenció que los deseos del usuario no representan en forma directa los requerimientos de primer nivel sino que muchos de ellos son derivados de otros. Esta característica se pudo verificar en la evaluación de la matriz de diseño, en donde el acople de los requerimientos era indicador de una consideración no adecuada.

Es necesario trabajar en la identificación de contradicciones y la traducción de necesidades del cliente en requerimientos, para proyectos de solución de necesidades sociales. La literatura actual abarca esencialmente problemas netamente técnicos en los que efectuar estos procesos resulta más evidente. Aunque durante el proyecto no se analizó el porqué de las dificultades en estas actividades, sí se evidenció que son tareas críticas dentro del proceso. 


\section{Referencias}

Aguilar-Zambrano, J. (2009). Ampliación del Modelo de Diseño Axiomático para el Desarrollo de Productos con Equipos Multidisciplinares. Tesis de doctorado, Universidad politécnica de Valencia, Valencia, España.

Aguilar-Zambrano, J., Gonzalez Cruz, M. C. \&. Aguilar-Zambrano, J.J. (2009). Un modelo de convergencia Empresa-Usuario-Universidad a partir de un Modelo Ampliado del Diseño Axiomático. Proceedings de ALTEC: XIII Seminario Latino-Iberoamericano de Gestión

Tecnológica ISBN: 978-958-8387-32-I p.75

Asociación Española de Normalización y certificación [AENOR] (2007). Productos de apoyo para personas en situación de discapacidad. Clasificación y terminología (ISO9999:2007). Madrid, España: Autor.

CavalluccI, D. \& Weill, R.D. (2001).Integrating Altshuller's development laws for technical systems into the design process. CIRP Annals.Manufacturing Technology, 50(1), pp. 115-120.

Saaty, T. (1994).Fundamentals of decision making and priority theory with the AHP.RWS Publications, Pittsburgh.

Salamatov, Y. (1999). TRIZ:The right solution at the right time. The Netherlands: Insytec B.V.

Savransky S. (2000). Engineering of Creativity. (first edition), Boca Raton, USA: CRC Press.

Shen, S.-T., Prior, S. D., White, A. S., and Karamanoglu, M. (2007). Using personality type diferences to form engineering design teams. Engineering education, 2(2):54-67.

Suh, N. P. (1990). Principles of Design. Oxford University, firstedition.

Recibido: septiembre 10 / Aprobado: diciembre 1 de 2012 